\title{
BMJ Open Effects of complex interventions in 'skin cancer prevention and treatment': protocol for a mixed-method systematic review with qualitative comparative analysis
}

Karolina Beifus, ${ }^{1}$ Eckhard Breitbart, ${ }^{2}$ Juliane Köberlein-Neu ${ }^{1}$

To cite: Beifus $\mathrm{K}$, Breitbart $\mathrm{E}$, Köberlein-Neu J. Effects of complex interventions in 'skin cancer prevention and treatment': protocol for a mixed-method systematic review with qualitative comparative analysis. BMJ Open 2017;7:e017196. doi:10.1136/ bmjopen-2017-017196

- Prepublication history for this paper is available online. To view these files please visit the journal online (http://dx.doi. org/10.1136/bmjopen-2017017196).

Received 8 May 2017 Revised 29 June 2017 Accepted 11 July 2017

CrossMark

${ }^{1}$ Center for Health Economics and Health Services Research, University of Wuppertal, Wuppertal, North RhineWestphalia, Germany

${ }^{2}$ Association of Dermatological Prevention, Buxtehude, Germany

Correspondence to

Karolina Beifus;

beifus@wiwi.uni-wuppertal.de

\section{ABSTRACT}

Introduction Occurring from ultraviolet radiation combined with impairing ozone levels, uncritical sun exposure and use of tanning beds an increasing number of people are affected by different types of skin cancer. But preventive interventions like skin cancer screening are still missing the evidence for effectiveness and therefore are criticised. Fundamental for an appropriate course of action is to approach the defined parameters as measures for effectiveness critically. A prerequisite should be the critical application of used parameter that are defined as measures for effectiveness. This research seeks to establish, through the available literature, the effects and conditions that prove the effectiveness of prevention strategies in skin cancer.

Method and analysis A mixed-method approach is employed to combine quantitative to qualitative methods and answer what effects can display effectiveness considering time horizon, perspective and organisational level and what are essential and sufficient conditions to prove effectiveness and cost-effectiveness in skin cancer prevention strategies. A systematic review will be performed to spot studies from any design and assess the data quantitatively and qualitatively. Included studies from each key question will be summarised by characteristics like population, intervention, comparison, outcomes, study design, endpoints, effect estimator and so on. Beside statistical relevancies for a systematic review the qualitative method of qualitative comparative analysis (QCA) will be performed. The estimated outcomes from this review and QCA are the accomplishment and absence of effects that are appropriate for application in effectiveness assessments and further cost-effectiveness assessment. Ethics and dissemination Formal ethical approval is not required as primary data will not be collected.

Trial registration number International Prospective Register for Systematic Reviews number CRD42017053859.

\section{BACKGROUND}

Skin cancer is an increasing health risk factor all over the world. Occurring from ultraviolet (UV) radiation combined with impairing
Strengths and limitations of this study

- This approach accomplishes the opportunity to complement a qualitative method to evidencebased medicine efforts and combine outcomes from different study designs.

- This study will contribute to frame appropriate effect parameters to use for assessments of effectiveness and cost-effectiveness and therefore prove these.

- This protocol is written following the published Preferred Reporting Items for Systematic Review and Meta-analysis Protocols guidelines.

- The qualitative comparative analysis is dependent to the quantity of appropriate and included studies.

ozone levels, uncritical sun exposure and use of tanning beds and increasing number of people are affected by different types of skin cancer. The incidences of non-melanoma skin cancer account for 2-3 million and for malignant melanoma 132000 annually worldwide. ${ }^{1}$ The burdens of illness in melanoma and non-melanoma skin cancer are multifaceted and affect sick persons, family members, as well as the society and furthermore governmental institutions as responsible instance for all individuals in a country.

By causing slight symptoms in the early stages, persons suffering from skin cancer often run into high stages of illness. Delayed medical attention in higher stages demand a more invasive and cost-intensive therapy. Also, patients undergo losses in their quality of life from symptoms and invasive therapy. Accompanied losses of income from absenteeism in patients as well as in their caregiving family members appear. Despite continually increasing incidences in skin cancer entities like malignant melanoma (MM), basal cell carcinoma (BCC) and squamous cell carcinoma (SCC) most people are still 
misunderstanding the hazard from UV radiation from sun or tanning beds. The use of sunbeds has been identified as the most significant risk increasing factor of melanoma and non-melanoma skin cancer. ${ }^{2}$ Over $65 \%$ of whites aged 18-29 years reported at least one sunburn in the past 12 months in the USA. ${ }^{3}$

Prevention strategies in each stage, primary, secondary and tertiary are in high demand. But interventions like skin cancer screening are still missing the evidence for effectiveness and therefore are criticised. A prerequisite should be, however, the critical application of the defined parameters as measures for effectiveness.

In the current literature, there is no distinct evidence for the effectiveness of skin cancer screening for example. Endpoints like mortality and morbidity increases are employed to prove the effectiveness of screening. But endpoints like these need an adjusted view on the stage of prevention, a corresponding time horizon, the perspective of account and so on.

Still studies rated in high quality by evidence-based medicine like randomised clinical trials (RCT), observational studies, ecological studies were taken into consideration. The recent issue is the report from the US Preventive Services Task Force by Wernli $e t a l{ }^{4}$

Certainly, prevention interventions have to be characterised as complex interventions. The Medical Research Council defines some key features for complex interventions as:

- Number of interacting components;

- Number and difficulty of behaviours required by those delivering or receiving the intervention;

- Number of groups or organisational levels targeted by the intervention;

- Number and variability of outcomes;

- Non-pharmacological;

- Behavioural;

- Lack of linear, well-evidenced causal pathways linking between interventions and the health outcomes as well as

- Feedback loops, synergies and phase changes. ${ }^{5}$

All interventions of prevention primary, secondary and tertiary can be classified as complex interventions by fulfilling all the mentioned key features. Therefore, they should be handled with approaches that include more study designs besides RCTs and similar controlled designs. To understand and evaluate complex interventions there is a need for quasiexperimental study designs, using control/comparison groups/areas and also uncontrolled studies for example, time series analysis, before-and-after studies and so on.

Further multiple players in the complex interventions of prevention are affecting the process and influencing the outcomes. The WHO defined the different participants and structures that are involved in the process of implementation, execution, maintenance and continuous modification of preventive and curative interventions within the technical brief 'integrated health services'. ${ }^{6}$ This approach is adopted to clarify the delivery of services or interventions and the acceptance or claim of interventions. The defined different levels such as the micro level for the 'user' or 'patient', the meso level for the 'provider' or 'professionals' and the macro level for 'policy-makers' by deciding, financing and regulating health services are employed. ${ }^{6}$ These different levels show the circumstances that can be understood as the 'context' for an intervention and will be henceforth be referred to as 'organisational levels'. Interventions are highly context sensitive and on the other side the context is complex and often poorly anticipated and accommodating to interventions. The 'double complexity' of intervention and context and their interaction is a central and important issue, which is essential and necessarily to be evaluated or at least mentioned. ${ }^{7}$ To exceed a broad outline of the organisational levels, each is examined in several dimensions. Therefore, Donabedian's framework for quality improvement ${ }^{8}$ is adduced. Structure, process and outcome can be evaluated on that base. Structure is defined by permanent capabilities of provider and requirement of user. The process covers all activities of each participant in the intervention, and the outcome concerns all results from produced and demanded performances.

Against this background, an analytical framework has been conceptualised which is examining the diversity of the structures in complex interventions of prevention and also interactions in the context. Research questions are conceived for different levels. Divers expected and factual effects as well as outcomes are implemented in this framework.

\section{Relevance of review}

This review seeks to outline effects of medical, social, communicative and economical aspects relevant for complex interventions in prevention in different skin cancer stages with the understanding of health services research.

Based on two scientifically established theories, the natural history of disease and the steps of prevention, this framework (figure 1) provides a working basis to point out key questions for the review. The effects and outcomes in the different levels with concomitant interactions can be displayed graphically.

In this framework three main issues are in focus:

1. Prevention stage: each stage of prevention implicates specific interventions. In secondary prevention for example, screening can be provided. In primary prevention entirely different approaches have to be utilised.

2. Time horizon: time progression figures into several aspects, which are reciprocally combined. So the time horizon has to be considered in natural history of disease and also in proceeding prevention stages from primary to tertiary. The choice of time frame or point in time has high impact on the effects of prevention such as overdiagnosis in screenings for example.

3. Participants and structure in the process of prevention: patients, professionals and in the end the whole society have to be included in the evaluation process of complex interventions. These players have influence 


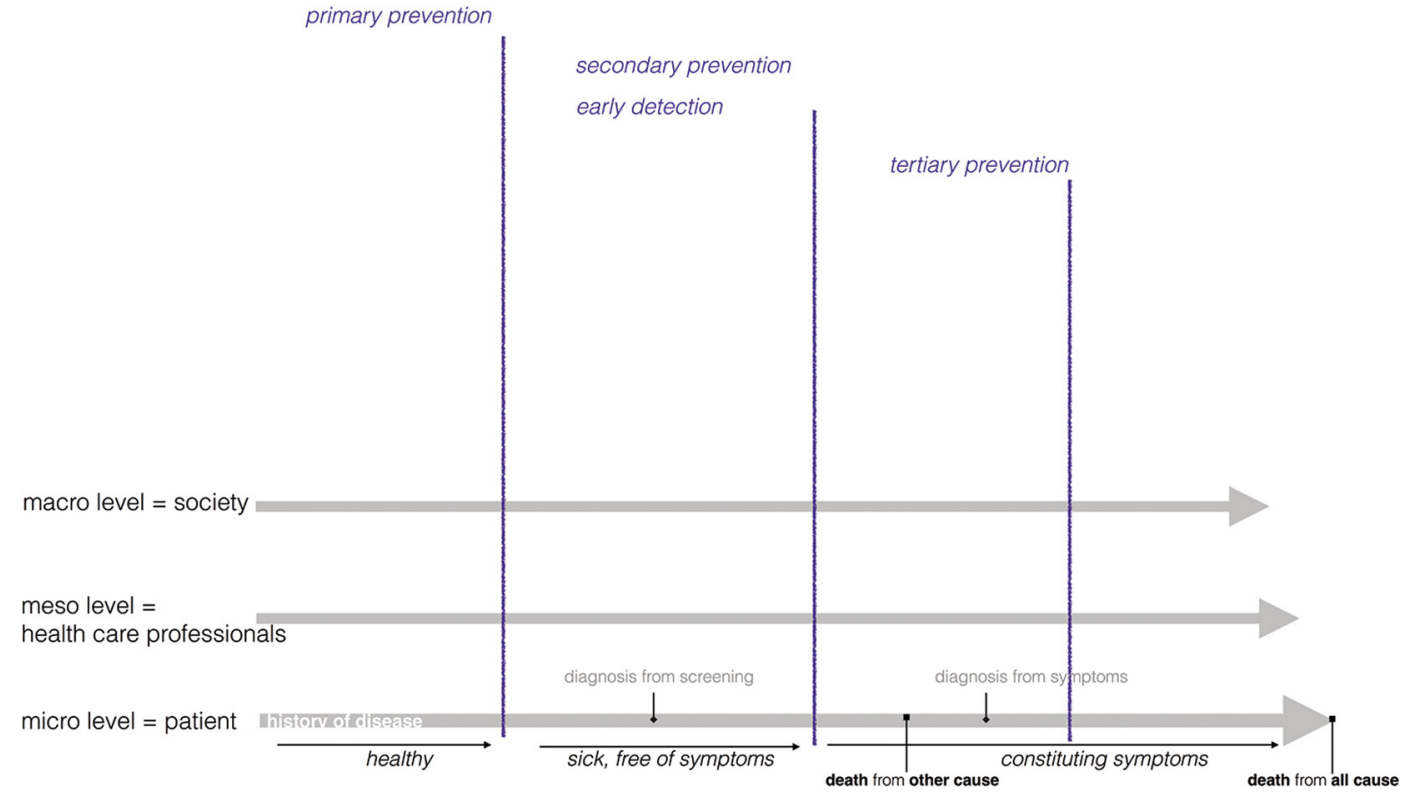

Figure 1 Analytical framework.

with their behaviours, decisions, skills, and so on and consequently influence the given context for complex interventions where they have to operate within. These participants and their specific perspective implicate divergent aspects of effects (benefits and costs) which all have to be considered individually. The participants and their perspectives are displayed in the different 'organisational levels' (macro, meso and micro) based on the WHO working definition on integrated health services. ${ }^{6}$ Within each level, the structure, process and outcome that result from interactions are considered.

\section{METHOD}

\section{Objective and key questions}

This review seeks to establish, through the available literature, the effects and conditions that prove the effectiveness of prevention strategies in skin cancer. The review protocol is authored according to PRIMA-P reporting guideline $^{9}$ and registered in Prospective Register for Systematic Reviews with the registration number CRD42017053859.

The specific research questions to be addressed are:

Key question 1: Aspects of effects and effectiveness

What effects can display effectiveness considering time horizon, perspective and organisational level?

a. What interventions against skin cancer have been realised?

b. What effects have been reported from skin cancer prevention strategies?

c. What effects and outcomes have been assigned in each organisational level in structure, process, and outcome?

d. What time horizon has been declared for the effects?

e. Is the interaction between intervention and context considered? f. What are essential and sufficient conditions to reach the effect that was reported?

g. What reasons hampered the achievement of the requested effect?

Key question 2: Aspects of costs and effectiveness

What are essential and sufficient conditions to prove effectiveness and cost-effectiveness in skin cancer prevention strategies?

a. What health economic analyses have been performed for primary, secondary, and tertiary prevention up to now?

b. What health economic aspects have been evaluated?

c. What are reported endpoints for effectiveness from skin cancer prevention and what time horizon do they require to prove cost-effectiveness?

Each key question considers the tumour entities malignant melanoma, BCC, and SCC. The key questions will be approached in particular systematic reviews.

\section{Searching strategy}

For all key questions the search will be conducted in following databases:

- PubMed (including Medline)

- PMC via PubMed

- PubMed Health

- EMBASE

- NHSEED

- CIN AHL

$\checkmark$ CRD

- DARE

- PsycInfo

- Scopus.

Following searching terms (figure 2) will be employed for complex 1:

In complex two applied search terms (figure 3): 


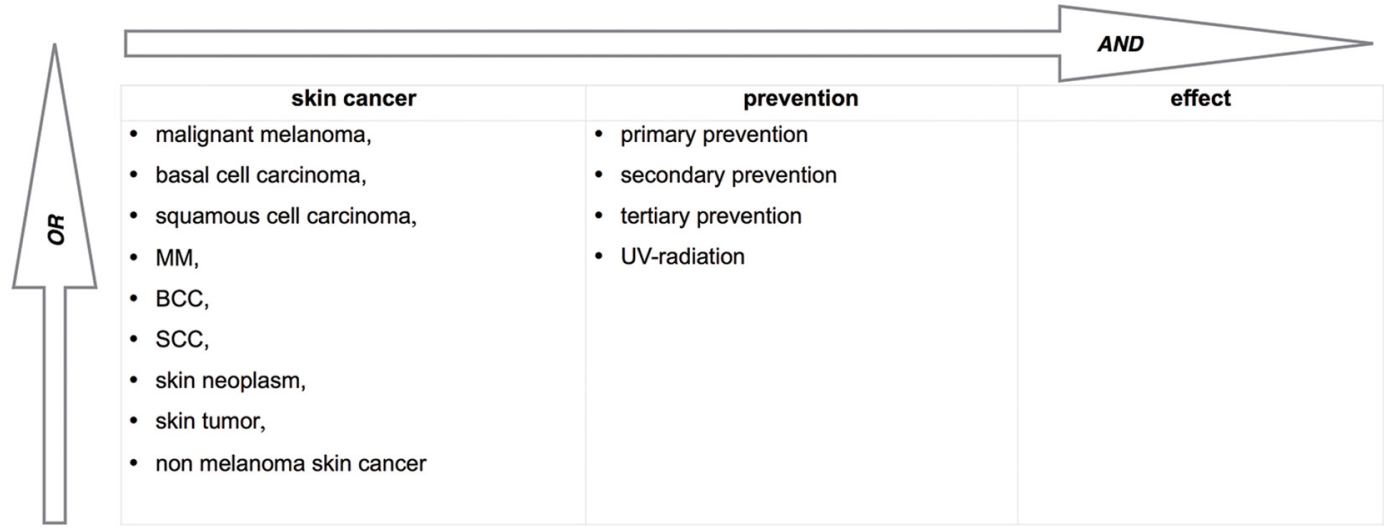

Figure 2 Searching terms for key question 1.

Listing all relevant terms in hierarchical form performed the selection of keywords for the electronic search. All synonyms, alternative terminology, related terms, word-stems, truncation, abbreviations, and acronyms were checked and included.

For databases a keyword search and phrases search in title and abstract fields is performed using the particular thesaurus option (eg, MeSH, EMTREE,etc.).

To access grey literature international databases will be examined. PhD theses and dissertations, current trials, and conference proceedings are searched for the databases Health Management Information Centre (Ovid) and Global Health (Ovid), Scopus, Web of Science. Further the search repositories www.greylit.org, Open Grey, and GreyNet International are browsed. An additional handsearch is performed in Google Scholar as well as reference lists in the included literature.

The key questions are processed within the PICOschema (figure 4). Using aspects for the evaluation in health economics according to German Institute for Quality and Efficiency in Healthcare the PICO-schema is extended. ${ }^{10}$

\section{Inclusion and exclusion criteria}

Titles and abstracts will be screened for eligibility according to the following inclusion criteria:

- international studies,

- language English and German,

- human relation, studies with any design dealing with primary, secondary, and tertiary prevention of skin cancer,

- grey literature,

- studies up to 40 years retrospective,

- studies with any design containing information on effects (quantitative and qualitative) from prevention interventions,

- quality of life in patients suffering from MM, BCC, or SCC,

- quality of life affected by therapy options against MM, BCC, SCC,

$\checkmark$ costs.

Exclusion criteria are denoted by:

- studies in other languages than German or English,

- other cancer entities than skin cancer,

- studies dealing with treatment and behaviour in other primary disease than skin cancer,

- studies only dealing with effects from pharmaceutical agent tests,

- duplicates,

- systematic reviews,

- meta analysis.

Data management and selection process

Articles identified through reference lists in included studies, grey literature, and bibliographic searches will also be considered for data collection based on

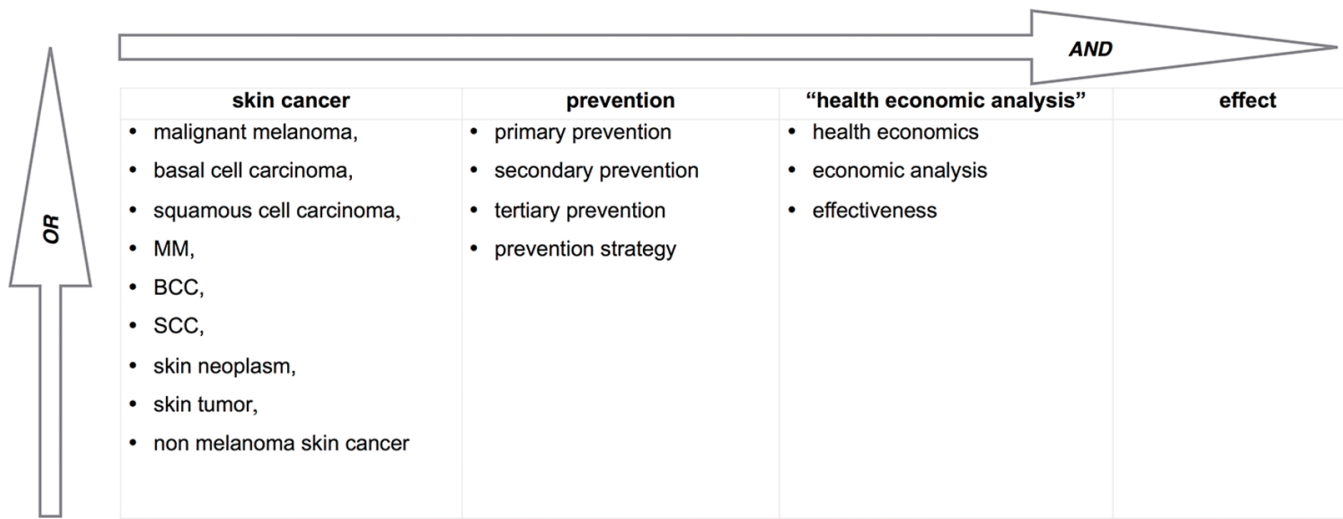

Figure 3 Searching terms for key question 2. 


\section{Population $\quad$ : whole population \\ - persons at risk \\ - age-specific perspective}

Intervention

Comparison

Outcomes

Economics
- self-examination

- whole body examination

- partial surfaces examined

- population based program

- information dissemination

- whole population vs. persons at risk

- self-exam. vs. population based screening

- age-specific vs. whole population
- saved persons

- detected cases

- costs

- false positives

- false negative

- cost-effectiveness

- cost-benefit

- cost-of-illness

\section{Health System • geographical relatedness \\ - health care system}

Figure 4 Examples for issues in an extended PICO-schema.

their title, abstract, and full text. Two reviewers will independently select articles regarding the inclusion criteria. Disagreements in reviewer selections will be resolved at a meeting between reviewers prior to selected articles being retrieved. Established tools for quality assessment of included studies are not completely suitable in the applied approach because of diversity of study designs. Therefore studies will be assessed by the reviewer with tool for quality of reporting corresponding to study design. In this expectation checklists CHEERS, ${ }^{11}$ CONSORT, ${ }^{12}$ ENTREQ,${ }^{13}$ $\mathrm{STaRI}^{14}$ and $\mathrm{STROBE}^{15}$ will be applied. The inter-rater reliability between the two will be assessed. ${ }^{16}$ Included studies from each key question will be summarised by characteristics like population, intervention, comparison, outcomes, study design, context characteristics, endpoints, effect estimator, etc. Studies will be categorised into affiliation to prevention level. The process of literature research will be displayed following the Preferred Reporting Items for Systematic Review and Meta-analysis flowchart. ${ }^{17}$ Study collection and assorting will be performed in BibTex V.0.99d.

\section{Data synthesis}

Statistical analysis

Included studies will be summarised by study design to achieve statistical outcomes from systematic review. If possible all effect estimators like ORs, relative risks, number needed to treat, risk reduction, costs and so on will be extracted pooled. Depending on heterogeneity, a fixed or random effect model will be used. The determination of heterogeneity will be tested with $\mathrm{I}^{2}$ statistics.

For statistical analysis STATA V.12 (StataCorp) will be employed.

Anyway, all outcomes will be reported in a narrative way.

\section{Qualitative analysis}

Furthermore a qualitative analysis will be conducted within a qualitative comparative analysis (QCA).

QCA is used for the purpose of methodological advantages:

- Complex causalities are an underlying assumption;

- A cross-case comparison: studies from review of each design and quality can be compared by contents and outcomes; 
- Each study is treated as a 'case' in QCA and brings along a combination of factors (characteristics of study as describes ahead); the combination of factors is called 'conditions';

- Conditions produce outcomes or they do not (both results are provable);

- The synergy of the conditions is a pivotal component of the QCA = 'conjunctural causation';

- QCA captures the assumption that multiple paths may coexist to a desired outcome = 'equifinality';

- Occurrence of outcomes has another reason than their absence = 'asymmetric causation'. ${ }^{18} 19$

Following working steps will be applied for the QCA in this review:

1. Prepare data characteristics tables;

2. Creating a truth table for a crips set QCA;

3. Evaluation of essential and sufficient conditions by using Boolean and Quine-McClusky algorithmsanalysis to check on consistency (degree to which combinations in the studies induce outcomes) and coverage (proportion of cases with an desired outcome);

4. Crisp truth table aids a fuzzy set analysis;

5. Fuzzy set analysis will be used to evaluate the degree to which each study answers the question on essential and sufficient conditions for the evidence of effectiveness from reported outcomes.

QCA will be performed with the software R.

\section{Dealing with protocol amendments}

Divergences from the protocol in the ongoing review process will be registered and documented for appearance, estimated reason and resolving strategy. This documentation will be reported in the respective review publication for the key questions.

\section{DISCUSSION}

The applied quantitative and qualitative methods and expected outcomes offer an appropriate method to reveal experienced interventions, their context and effects.

By extending the purpose of evidence-based medicine by qualitative efforts with regard to the underlying conditions for the attainability of effects and their absence, this presents an indispensable groundwork to frame all suitable endpoints for effectiveness measures and furthermore cost-effectiveness.

Acknowledgements This work has been funded by the Association of Dermatological Prevention (ADP) kindly supported by professionally qualified help of $\mathrm{EB}$ and Susanne Fengler from the ADP in Buxtehude.

Contributors KB conceived the idea, the literature review, evaluation design and preparation of this study protocol. JK-N contributed to refinement of the study protocol and approved the final manuscript. The accomplishment of literature review, selection and quality assessment resides by KB and JK-N. Statistical expertise and qualitative comparative analysis are planned and performed by KB. This work has been funded by the Association of Dermatological Prevention (ADP) kindly supported by professionally qualified help of EB.
Funding The ADP with supervision by Prof Eckhard Breitbart is funding the systematic literature research with QCA, which is sponsored by the LEO Foundation Denmark for superior research in skin cancer prevention.

Competing interests None declared.

Provenance and peer review Not commissioned; externally peer reviewed.

Open Access This is an Open Access article distributed in accordance with the Creative Commons Attribution Non Commercial (CC BY-NC 4.0) license, which permits others to distribute, remix, adapt, build upon this work non-commercially, and license their derivative works on different terms, provided the original work is properly cited and the use is non-commercial. See: http://creativecommons.org/ licenses/by-nc/4.0/

(c) Article author(s) (or their employer(s) unless otherwise stated in the text of the article) 2017. All rights reserved. No commercial use is permitted unless otherwise expressly granted.

\section{REFERENCES}

1. World Health Organization. Ultraviolet radiation and the INTERSUN Programme - How common is skin cancer? 2016. http://www.who. int/uv/faq/skincancer/en/index1.html

2. Boniol $\mathrm{M}$, Autier $\mathrm{P}$, Boyle $\mathrm{P}$, et al. Cutaneous melanoma attributable to sunbed use: systematic review and meta-analysis. BMJ 2012;345:e4757.

3. Centers for Disease Control and Prevention. Sunburn and sun protective behaviors among adults aged 18-29 years-United States, 2000-2010. MMWR - Mortality and morbidity Weekly Report 11:31722.

4. Wernli KJ, Henrikson NB, Morrison CC, et al. Screening for skin cancer in adults: updated evidence report and systematic review for the US preventive services task force. JAMA 2016;316:436-47.

5. Craig P, Dieppe P, Macintyre S, et al. Developing and evaluating complex interventions: the new Medical Research Council guidance. BMJ 2008;337:a1655

6. World Health Organization. Integrated health services - what and why? Technical brief No. 1, 2008.

7. Schrappe M, Pfaff $H$. Versorgungsforschung vor neuen Herausforderungen: konsequenzen für definition und Konzept. Gesundheitswesen 2016;78:689-94.

8. Donabedian A. The criteria and standards of quality. Ann Arbor, Mich.: Health Administration Press, 1982.

9. Shamseer L, Moher D, Clarke M, et al. Preferred reporting items for systematic review and meta-analysis protocols (PRISMA-P) 2015: elaboration and explanation. BMJ 2015;349:g7647.

10. Droste S, Dintsios C-M. Literature searching for Publications used in Economic evaluation in Health Technology Assessments. Gesundh ökon Qual manag2011;16:35-57.

11. Husereau D, Drummond M, Petrou S, et al. Consolidated Health Economic evaluation Reporting Standards (CHEERS) statement. BMJ 2013;346:f1049.

12. Moher D, Schulz KF, Altman DG. The CONSORT statement: revised recommendations for improving the quality of reports of parallel-group randomised trials. The Lancet 2001;357:1191-4.

13. Tong A, Flemming K, Mclnnes E, et al. Enhancing transparency in reporting the synthesis of qualitative research: ENTREQ. BMC Med Res Methodol 2012;12:181

14. Pinnock H, Barwick M, Carpenter CR, et al. Standards for Reporting Implementation studies (StaRI) Statement. BMJ 2017;356:i6795

15. Vandenbroucke JP , et al. Strengthening the Reporting of Observational studies in Epidemiology (STROBE): explanation and elaboration. Ann Intern Med 2007;147. W.

16. Landis JR, Koch GG. The measurement of observer agreement for categorical data. Biometrics 1977;33:159-74.

17. Moher D, Liberati A, Tetzlaff J, et al. Preferred reporting items for systematic reviews and meta-analyses: the PRISMA statement. PLOS Med 2009;6:e1000097.

18. Raign CC. The comparative method: moving beyond qualitative and quantitative strategies: university of California Press, 1987.

19. Rihoux B. Configurational comparative methods: qualitative comparative analysis (QCA) and related techniques. Los Angeles CA u.a: Sage Publ, 2009. (Applied social research methods series; vol 51). 